ARTIGO ORIGINAL ORIGINAL ARTICLE
Palavras-chave:

neoplasias pulmonares, análise custo-benefício, afatinibe

\section{Custo-efetividade do afatinibe versus pemetrexede associado a cisplatina, erlotinibe e gefitinibe no tratamento de primeira linha de pacientes com câncer de pulmão não pequenas células localmente avançado ou metastático, com mutação do receptor do fator de crescimento epidermoide (EGFR+), na perspectiva do Sistema de Saúde Suplementar do Brasil}

\author{
Cost-effectiveness of afatinib versus pemetrexed plus \\ cisplatin, erlotinib and gefitinib as first line treatment in \\ patients with locally advanced or metastatic epidermal \\ growth factor receptor mutation (EFGR+) non-small cell \\ lung cancer in the Brazilian private healthcare system
}

Natália Bolzachini Santoni', Thaís Gomes de Melo', Anna Rita Aguirre', Daniela Luciano Pastor Veiga', Camila Pepe Ribeiro de Souza²

DOI: 10.21115/JBES.v9.n1.p73-82

\section{RESUMO}

Objetivo: Comparar os custos e efetividade do afatinibe versus pemetrexede associado a cisplatina (PEM/CIS), erlotinibe e gefitinibe no tratamento de primeira linha de pacientes com câncer de pulmão não pequenas células (CPNPC) com mutação no receptor de fator de crescimento epidermoide (EGFR+) localmente avançado ou metastático, no Sistema de Saúde Suplementar brasileiro. Métodos: $O$ modelo de Markov foi utilizado para estimar anos de vida livres de progressão (PFLY), anos de vida (LY), anos de vida ajustados pela qualidade (QALY) e desfechos clínicos por sete anos. Utilizaram-se dados de sobrevida, segurança e utilidade dos estudos LUX-Lung 1, 3 e 6 e LUCEOR. A eficácia comparativa versus gefitinibe e erlotinibe foi estimada utilizando modelos bayesianos de comparação indireta. A utilização dos recursos foi estimada por painel de especialistas, e custos diretos foram estimados utilizando-se bases de dados oficiais. Resultados: Afatinibe mostrou aumento da sobrevida livre de progressão (0,41 PFLY), sobrevida global $(0,16 \mathrm{LY})$ e qualidade de vida (0,21 QALY) com custo incremental (R\$ 8.549), resultando em razão de custo-efetividade incremental (RCEI) de R\$20.639/PFLY. Comparado ao erlotinibe, o afatinibe mostrou aumento de 0,46 PFLY, 0,13 LY e 0,20 QALY, com menor custo (-R\$ 21.327). Comparado ao gefitinibe, o afatinibe mostrou incrementos de 0,53 PFLY, 0,37 LY, 0,34 QALY, com custo incremental de R\$24.890, resultando em RCEI de R\$ 46.709/PFLY. Considerando-se três vezes o PIB per capita como limiar de custo-efetividade (R\$ 86.628), o afatinibe é custo-efetivo versus PEM/CIS e gefitinibe e dominante quando comparado ao erlotinibe. Conclusão: Sugere-se que o afatinibe é uma opção custo-efetiva quando comparado ao PEM/CIS, erlotinibe e gefitinibe no tratamento de primeira linha de pacientes com CPNPC EGFR+.

Recebido em: 02/03/2017. Aprovado para publicação em: 01/04/2017

1. Boehringer Ingelheim, São Paulo, SP, Brasil.

2. Sense Company, São Paulo, SP, Brasil.

Instituição onde o trabalho foi executado: Sense Company, São Paulo, SP, Brasil.

Informações sobre auxílios recebidos sob a forma de financiamento, equipamentos ou medicamentos: Este projeto foi financiado pela Boehringer Ingelheim do Brasil.

Congressos onde o estudo foi apresentado: Este estudo foi aceito no Congresso "ISPOR 22nd Annual International Meeting", 2017.

Autor correspondente: Natália Bolzachini Santoni. Av. das Nações Unidas, 14171, $18^{\circ}$ andar - Vila Gertrudes, São Paulo/SP _

04794-000. Telefone: +55 (11) 4949-4955. E-mail: natalia.santoni@boehringer-ingelheim.com 


\section{Keywords:}

lung neoplasms, costbenefit analysis, afatinib

\begin{abstract}
Objective: To compare costs and effectiveness of afatinib versus pemetrexed plus cisplatin (PEM/ CIS), erlotinib and gefitinib, as first line treatment in patients with locally advanced or metastatic epidermal growth factor receptor mutation (EGFR+) non-small cell lung cancer (NSCLC) in the Brazilian Private Healthcare System. Methods: A Markov model was used to estimate 7 year progression-free life years (PFLY), life years (LY), quality-adjusted life years (QALY) and clinical outcomes of afatinib. Partitioned survival, safety and utility data from the LUX-Lung 1, 3 and 6 and LUCEOR trials were used. Comparative effectiveness versus gefitinib and erlotinib was estimated using Bayesian indirect treatment comparison. Resource use was estimated by an expert panel and direct costs were estimated from official databases. Results: Compared with PEM/CIS, afatinib was associated with increased progression free survival (0.41 PFLY), increased overall survival ( $0.16 \mathrm{LY})$ and increased quality of life (0.21 QALY) with incremental cost (BRL 8,549), resulting in an incremental cost-effectiveness ratio (ICER) of BRL 20.639/PFLY. Compared to erlotinib, afatinib was associated with additional 0.46 PFLY, $0.13 \mathrm{LY}$ and $0.20 \mathrm{QALYS}$ with lower cost (-BRL 21,327). When compared to gefitinib, afatinib was associated with incremental 0.53 PFLY, 0.37 LY and 0.34 QALY and increased cost (BRL 24,890), resulting in an ICER of BRL 46,709/PFLY. Considering 3 PIB per capita as a threshold (BRL 86,628), afatinib is a cost-effective technology versus PEM/CIS and gefitinib and dominant when compared to erlotinib. Conclusion: Findings suggest that afatinib is a cost-effective option, when compared to PEM/CIS, erlotinib and gefitinib, as first line treatment in EGFR+ NSCLC patients.
\end{abstract}

\section{Introdução}

O câncer de pulmão representa a causa mais importante de óbito por câncer no mundo e é considerado uma das principais causas de mortes evitáveis. No Brasil, em 2013, o número de mortes por esse tipo de câncer atingiu 24.490 pessoas, sendo 14.811 homens e 9.675 mulheres. Em 2016, estimam-se 17.330 casos novos de câncer de traqueia, brônquios e pulmões entre homens e 10.890 entre mulheres [Ministério da Saúde (Brasil). Instituto Nacional de Câncer José Alencar Gomes da Silva (Inca), 2015].

Para fins terapêuticos e prognósticos, o câncer de pulmão é agrupado, segundo o tipo histopatológico, em câncer de pulmão de pequenas células (CPCP) e câncer de pulmão não pequenas células (CPNPC), este último responsável por quase 90\% de todos os casos [Ministério da Saúde (Brasil). Secretaria de Atenção à Saúde, 2014].

Além do diagnóstico histológico, outras classificações tumorais, como o perfil molecular, são fundamentais para a definição do tratamento ideal, uma vez que cada tipo de mutação se comporta como uma doença diferente (Dietel et al., 2015).

Muitos pacientes com CPNPC possuem mutações no receptor do fator de crescimento epidermoide (EGFR), identificado como importante causador da proliferação celular no CPNPC. As mutações mais frequentes são a deleção do éxon 19 e a substituição de leucina por arginina na posição 858 do éxon 21 (L858R) (Hirsh, 2015). A superexpressão do EGFR não está relacionada ao tabagismo e está associada a um pior prognóstico no CPNPC (Jänne \& Johnson, 2006).

A doença é geralmente diagnosticada já em estágios avançados, por ter pouca sintomatologia nos estágios iniciais [Ministério da Saúde (Brasil). Instituto Nacional de Câncer José de Alencar Gomes da Silva (Inca), 2014].
O impacto econômico do CPNPC é significativo. A intensificação da prevenção e uso de novas terapias pode reduzir o uso de recursos e os custos do tratamento. A falha no tratamento inicial do CPNPC está associada a significativos incrementos nos custos do manejo da doença.

O afatinibe é o representante de uma segunda geração de inibidores de tirosina quinase (TKI) com ação no EGFR, irreversível, com ação inibitória sobre toda a família ErbB, capaz de prolongar o tempo até a progressão da doença e a resposta ao tratamento. É indicado para o tratamento de pacientes adultos sem utilização prévia de EGFR-TKI, com CPNPC localmente avançado ou metastático com mutação do EGFR, como primeira linha de tratamento ("LUX-Lung 7: A Phase IIb Trial of Afatinib (BIBW2992) Versus Gefitinib for the Treatment of 1st Line EGFR Mutation Positive Adenocarcinoma of the Lung - Tabular View - ClinicalTrials. gov," n.d.; Park et al., 2015; Sequist et al., 2013; Soria et al., 2015; Wu et al., 2014; Yang et al., 2012; Yang et al., 2013; Yang et al., 2015).

O presente estudo tem como objetivo realizar uma avaliação econômica, comparando as diferentes estratégias medicamentosas no tratamento de CPNPC, sendo elas afatinibe, pemetrexede associado a cisplatina (pemetrexede/cisplatina), erlotinibe e gefitinibe, e avaliar se os custos adicionais gerados pelo uso de afatinibe, em comparação com os demais medicamentos utilizados, são justificados pelos ganhos clínicos observados.

\section{Métodos}

Foi realizada uma análise de custo-efetividade comparando o afatinibe a outras três alternativas para o tratamento de câncer de pulmão não pequenas células (CPNPC): a) pemetrexede/cisplatina, b) erlotinibe e c) gefitinibe. 


\section{População-alvo}

Pacientes adultos com CPNPC localmente avançado ou metastático com mutação do EGFR, sem tratamento prévio com inibidores da TKI com ação no EGFR.

\section{Perspectiva do estudo}

A perspectiva adotada foi a do Sistema de Saúde Suplementar do Brasil.

\section{Desfechos}

A análise contou com três desfechos, sendo eles: anos de vida salvos (sigla em inglês LY, life years), anos de vida livres de progressão (sigla em inglês PFLY, progression free life years) e anos de vida ajustados por qualidade (sigla em inglês QALY, quality adjusted life years). O desfecho principal utilizado na apresentação dos resultados e na análise de sensibilidade probabilística no caso base foi o PFLY.

As estratégias de tratamento foram comparadas por meio da razão de custo-efetividade incremental (RCEI), definida como a relação entre a diferença de custos dividida pela diferença de efetividade entre as duas alternativas de estratégia de tratamento.

\section{Estrutura do modelo}

Os modelos de Markov têm dois componentes: estrutura e parâmetros. A "estrutura" refere-se aos estados de saúde representados no modelo e as transições possíveis entre eles. Os "parâmetros" do modelo incluem os valores de probabilidades atribuídos às transições entre estados de saúde.

Para a estimativa dos custos e desfechos dos tratamentos, foi elaborado um modelo de Markov, que acompanhou pacientes com CPNPC por um horizonte temporal de sete anos. Esse horizonte foi selecionado, pois, a partir de sete anos, mais de $90 \%$ dos pacientes da coorte simulada já se encontram no estado de saúde relacionado à morte.

O modelo considerou a transição dos pacientes por diferentes estados de saúde (Figura 1). Nesse modelo, os pacientes iniciam o tratamento do CPNPC avançado ou metastático com mutação do EGFR no estado livre de progressão (LP).

Os pacientes no estado livre de progressão podem morrer ou ir para o estado de pós-progressão da doença (PD). Pacientes que progridem permanecem nesse estado ou migram para a morte. Assim que o paciente passa para o estado de pós-progressão da doença, inicia-se a segunda linha de tratamento, que apresenta um tempo predefinido e finito de tratamento, até uma nova progressão da doença. Os pacientes que atingem o tempo-limite de tratamento em segunda linha passam imediatamente para o melhor cuidado de suporte (sigla em inglês BSC, best supportive care).

No modelo, a duração de cada ciclo de tratamento é de um mês, o que permite uma avaliação adequada da progressão da doença e sobrevida dos pacientes, uma vez que

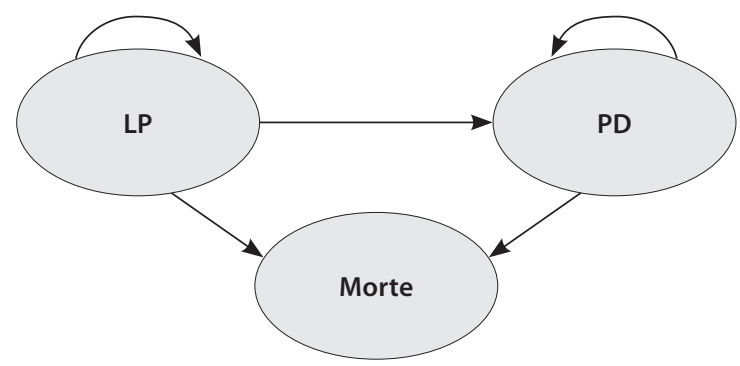

LP: livre de progressão; PD: progressão da doença.

Figura 1. Modelo de Markov.

é muito pequena a chance de o paciente progredir mais de uma vez dentro desse horizonte de tempo.

\section{Dados de eficácia clínica}

Para a comparação de afatinibe com pemetrexede/cisplatina, os dados foram extraídos dos estudos LUX-Lung 3 e LUX-Lung 6 (Wu et al., 2014; Yang et al., 2013), enquanto para a comparação com erlotinibe e gefitinibe os dados foram retirados de uma metanálise de comparação indireta (Popat et al., 2014; Wu et al., 2014; Yang et al., 2013).

Para calcular um período de sobrevida maior do que os considerados nos estudos clínicos, foram estimados modelos de sobrevida paramétricos, a partir dos dados obtidos das curvas de Kaplan-Meier dos ensaios clínicos. Para análise da sobrevida global e sobrevida livre de progressão, adotou-se uma curva de Weibull. As curvas de sobrevida livre de progressão, sobrevida global e seus hazard ratios (HR) para a comparação com pemetrexede/cisplatina são apresentadas na Figura 2. Com relação às comparações com erlotinibe e gefitinibe, as efetividades comparativas entre os grupos foram estimadas utilizando modelos bayesianos de comparação indireta e estão apresentados na Figura 3 e na Figura 4, respectivamente.

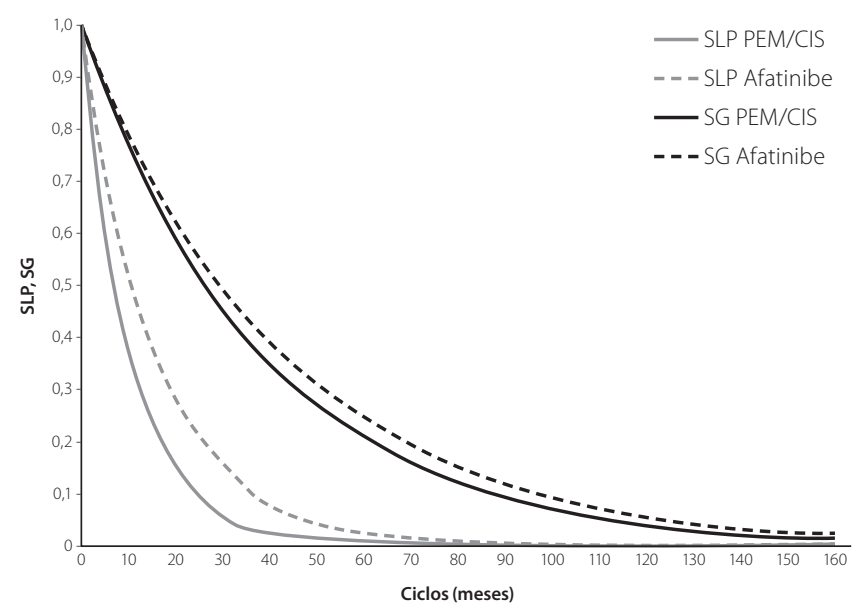

SLP: sobrevida livre de progressão; SG: sobrevida global; PEM/CIS: pemetrexede associado a cisplatina.

Figura 2. Afatinibe e pemetrexede/cisplatina - SLP e SG. 


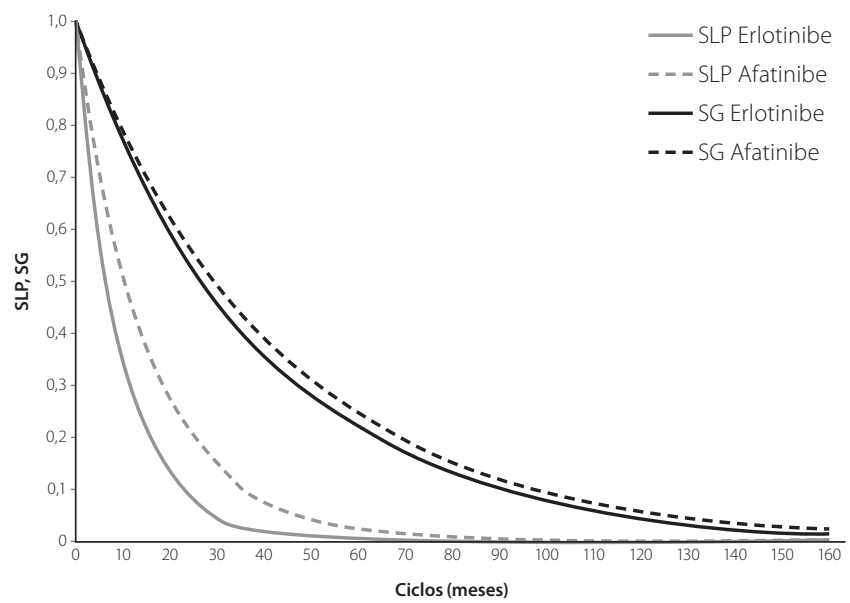

SLP: sobrevida livre de progressão; SG: sobrevida global.

Figura 3. Afatinibe e erlotinibe - SLP e SG.

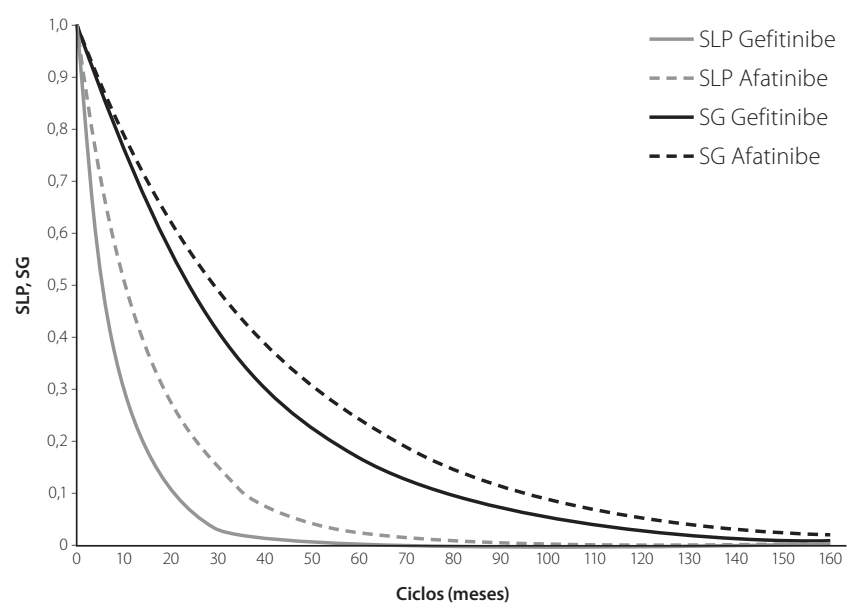

SLP: sobrevida livre de progressão; SG: sobrevida global.

Figura 4. Afatinibe e gefitinibe - SLP e SG.

A ocorrência de eventos adversos foi considerada apenas no estado livre de progressão, durante os tratamentos de primeira linha com afatinibe, pemetrexede/cisplatina, erlotinibe e gefitinibe, sendo considerados apenas os eventos de graus 3 e 4, retirados dos estudos LUX-Lung 1, LUX-Lung 3 e da metanálise de comparação indireta (Fleeman et al., 2010; Miller et al., 2012; Rosell et al., 2012; Yang et al., 2013; Zhou et al., 2011) A Tabela 1 apresenta a ocorrência de eventos adversos para cada tratamento presente na análise.

\section{Dados de custo}

Os desfechos econômicos contemplados foram os custos médicos diretos, que incluem os recursos médicos utilizados diretamente com o paciente. Custos indiretos e custos não médicos diretos não foram incluídos na análise.

Para determinação do uso de recursos para o tratamento e acompanhamento de eventos de CPNPC avançado ou metastático com mutação do EGFR, sem tratamento prévio com EGFR-TKI, utilizaram-se as recomendações das bulas dos medicamentos (AstraZeneca do Brasil Ltda., 2011; Boehringer Ingelheim do Brasil Quím. e Farm. Ltda., 2016; Produtos Roche Químicos e Farmacêuticos S.A., 2014), com exceção do padrão de tratamento com pemetrexede/cisplatina, que teve suas doses estabelecidas por meio da opinião de especialistas. A posologia considerada foi de um comprimido de 40 mg de afatinibe por dia, uma dose de 1.000 mg de pemetrexede e $200 \mathrm{mg}$ de cisplatina por ciclo de tratamento de três semanas (21 dias), um comprimido de 150 mg de erlotinibe por dia e um comprimido de $250 \mathrm{mg}$ de gefitinibe por dia.

A segunda linha de tratamento foi estimada de acordo com a opinião de especialistas. Após a progressão da doença com afatinibe, erlotinibe ou gefitinibe, considerou-se a utilização de pemetrexede em monoterapia para as análises. Já após a progressão com tratamento de pemetrexede/cisplatina, considerou-se como segunda linha de tratamento o erlotinibe.

Os valores de custos unitários dos medicamentos foram obtidos da lista de preços da Câmara de Regulação do Mercado de Medicamentos (CMED) de julho de 2016, considerando o Preço Fábrica (PF) com 18\% de ICMS. Como o erlotinibe apresenta isenção de ICMS, para ele se considerou o PF sem aplicação do imposto (Ministério da Saúde. Brasil, 2016). Os preços dos medicamentos utilizados na análise estão apresentados na Tabela 2.

As condutas adotadas para o manejo da doença nos estados livre de progressão e pós-progressão foram definidas e

Tabela 1. Ocorrência de eventos adversos

\begin{tabular}{lcccc}
\hline Evento adverso & Afatinibe & Pemetrexede/Cisplatina & Gefitinibe & Erlotinibe \\
\hline Diarreia & $14,4 \%^{\mathrm{A}}$ & $0,0 \%^{\mathrm{A}}$ & $3,6 \%^{\mathrm{C}}$ & $7,5 \%^{\mathrm{C}}$ \\
\hline Rash/Acne & $16,2 \%^{\mathrm{A}}$ & $0,0 \%^{\mathrm{A}}$ & $3,4 \%^{\mathrm{C}}$ & $16,0 \%^{\mathrm{C}}$ \\
\hline Fadiga & $1,3 \%^{\mathrm{A}}$ & $12,6 \%^{\mathrm{A}}$ & $5,2 \%^{\mathrm{C}}$ & $0,7 \%^{\mathrm{C}}$ \\
\hline Anemia & $0,4 \%^{\mathrm{A}}$ & $6,3 \%^{\mathrm{B}}$ & $0,4 \%^{\mathrm{A}}$ & $0,4 \%^{\mathrm{A}}$ \\
\hline Neutropenia & $0,4 \%^{\mathrm{A}}$ & $18,0 \%^{\mathrm{B}}$ & $0,4 \%^{\mathrm{A}}$ & $0,4 \%^{\mathrm{A}}$ \\
\hline Neutropenia febril & $0,0 \%^{\mathrm{D}}$ & $1,3 \%^{\mathrm{E}}$ & $0,0 \%^{\mathrm{D}}$ & $0,0 \%{ }^{\mathrm{D}}$ \\
\hline
\end{tabular}

A: Para afatinibe e pemetrexede/cisplatina, considerou-se o estudo LUX-Lung 3. E para gefitinibe e erlotinibe, assumiram-se as ocorrências do afatinibe (Yang et al., 2013). B: Zhou et al., 2011(Zhou et al., 2011). C: Metanálise de efeitos fixos. D: Para erlotinibe, considerou-se o estudo de Rosell et al., 2012 (Rosell et al., 2012 ). E para afatinibe e gefitinibe, considerou-se a ocorrência do erlotinibe. E: Estudo JMBD (Fleeman et al., 2010). 
Tabela 2. Custo unitário de medicamentos

\begin{tabular}{lcc}
\hline Medicamento & Unidade & Custo unitário \\
\hline Afatinibe & $40 \mathrm{mg}$ & $\mathrm{R} \$ 151,95$ \\
\hline Pemetrexede & $500 \mathrm{mg}$ & $\mathrm{R} \$ 6.915,76$ \\
\hline Cisplatina & $100 \mathrm{mg}$ & $\mathrm{R} \$ 251,65$ \\
\hline Erlotinibe & $150 \mathrm{mg}$ & $\mathrm{R} \$ 223,65$ \\
\hline Gefitinibe & $250 \mathrm{mg}$ & $\mathrm{R} \$ 132,03$ \\
\hline
\end{tabular}

validadas por especialistas, e os custos unitários foram retirados da Classificação Brasileira Hierarquizada de Procedimentos Médicos (CBHPM) e da Assistência à Saúde dos Servidores Públicos Estaduais (Planserv) [Associação Médica Brasileira (AMB), 2014]. Os custos anuais por estado de saúde estão apresentados na Tabela 3 e na Tabela 4.

Os custos de eventos adversos (EAs) também foram definidos por especialistas e custeados de acordo com as listas CMED, CBHPM e Planserv e estão apresentados na Tabela 5.

Tabela 3. Custo anual para pacientes livres de progressão

\begin{tabular}{|c|c|c|c|c|}
\hline Procedimentos ambulatoriais & $\%$ em uso & Quantidade & Custo unitário & Custo total \\
\hline Consulta com clínico geral & $100 \%$ & 2 & $\mathrm{R} \$ 71,68$ & $\mathrm{R} \$ 143,36$ \\
\hline Consulta com especialista & $100 \%$ & 6 & $\mathrm{R} \$ 71,68$ & $R \$ 430,08$ \\
\hline Consulta com enfermeiro & $100 \%$ & 3 & $R \$ 0,00$ & $R \$ 0,00$ \\
\hline Fisioterapia respiratória & $30 \%$ & 10 & $\mathrm{R} \$ 33,85$ & $R \$ 101,55$ \\
\hline Imunoistoquímica & $100 \%$ & 1 & $R \$ 467,04$ & $R \$ 467,04$ \\
\hline Hemograma & $100 \%$ & 4 & $R \$ 13,32$ & $R \$ 53,28$ \\
\hline Glicose & $100 \%$ & 4 & $R \$ 6,00$ & $R \$ 24,00$ \\
\hline Ureia & $100 \%$ & 4 & $\mathrm{R} \$ 6,00$ & $\mathrm{R} \$ 24,00$ \\
\hline Creatinina & $100 \%$ & 4 & $R \$ 6,00$ & $\mathrm{R} \$ 24,00$ \\
\hline Sódio & $100 \%$ & 4 & $R \$ 6,00$ & $R \$ 24,00$ \\
\hline Potássio & $100 \%$ & 4 & $\mathrm{R} \$ 6,00$ & $\mathrm{R} \$ 24,00$ \\
\hline Transaminase glutâmico-pirúvica (ALT) & $100 \%$ & 4 & $R \$ 11,04$ & $\mathrm{R} \$ 44,16$ \\
\hline Transaminase glutâmico-oxalacética (AST) & $100 \%$ & 4 & $R \$ 11,04$ & $R \$ 44,16$ \\
\hline Fosfatase alcalina & $100 \%$ & 4 & $R \$ 11,04$ & $R \$ 44,16$ \\
\hline Gama GT & $100 \%$ & 4 & $R \$ 11,04$ & $R \$ 44,16$ \\
\hline Bilirrubinas & $100 \%$ & 4 & $\mathrm{R} \$ 6,00$ & $\mathrm{R} \$ 24,00$ \\
\hline Tempo de protrombina & $50 \%$ & 4 & $\mathrm{R} \$ 8,73$ & $\mathrm{R} \$ 17,46$ \\
\hline Radiografia de tórax (posteroanterior e perfil) & $100 \%$ & 4 & $R \$ 51,74$ & $\mathrm{R} \$ 206,96$ \\
\hline Tomografia computadorizada de tórax & $100 \%$ & 2 & $\mathrm{R} \$ 519,72$ & $\mathrm{R} \$ 1.039,44$ \\
\hline Tomografia computadorizada de abdome & $100 \%$ & 2 & $\mathrm{R} \$ 791,27$ & $\mathrm{R} \$ 1.582,54$ \\
\hline Tomografia computadorizada de crânio & $100 \%$ & 1 & $\mathrm{R} \$ 459,18$ & $\mathrm{R} \$ 459,18$ \\
\hline RNM de tórax & $30 \%$ & 1 & $\mathrm{R} \$ 1.025,77$ & $\mathrm{R} \$ 307,73$ \\
\hline USG de tórax & $30 \%$ & 1 & $\mathrm{R} \$ 92,16$ & $R \$ 27,65$ \\
\hline Cintilografia óssea & $100 \%$ & 1 & $\mathrm{R} \$ 312,14$ & $\mathrm{R} \$ 312,14$ \\
\hline PET-TC & $30 \%$ & 1 & $\mathrm{R} \$ 969,13$ & $R \$ 290,74$ \\
\hline Procedimento cirúrgico & $15 \%$ & 1 & $\mathrm{R} \$ 8.415,59$ & $\mathrm{R} \$ 1.262,34$ \\
\hline Procedimentos intra-hospitalares & $\%$ uso & Quantidade & Custo unitário & Custo total \\
\hline Hospitalizações imprevistas & $10 \%$ & 10 & $\mathrm{R} \$ 447,94$ & $\mathrm{R} \$ 447,94$ \\
\hline Visitas à UTI & $5 \%$ & 10 & $\mathrm{R} \$ 1.817,50$ & $\mathrm{R} \$ 908,75$ \\
\hline Visitas à emergência & $10 \%$ & 10 & $R \$ 460,06$ & $R \$ 460,06$ \\
\hline TOTAL & & & & $\mathrm{R} \$ \mathbf{8 . 8 3 8 , 8 7}$ \\
\hline
\end{tabular}

RNM: ressonância nuclear magnética; USG: ultrassonografia; PET-TC: tomografia computadorizada por emissão de pósitrons; UTI: unidade de terapia intensiva. 
Tabela 4. Custo anual para pacientes pós-progressão da doença

\begin{tabular}{|c|c|c|c|c|}
\hline Procedimentos ambulatoriais & $\%$ uso & Quantidade & Custo unitário & Custo total \\
\hline Consulta com clínico geral & $100 \%$ & 4 & $R \$ 71,68$ & $\mathrm{R} \$ 286,72$ \\
\hline Consulta com especialista & $100 \%$ & 8 & $R \$ 71,68$ & $R \$ 573,44$ \\
\hline Fisioterapia motora & $40 \%$ & 6 & $\mathrm{R} \$ 27,18$ & $R \$ 65,23$ \\
\hline Fisioterapia respiratória & $50 \%$ & 8 & $R \$ 33,85$ & $R \$ 135,40$ \\
\hline Imunoistoquímica & $100 \%$ & 1 & $R \$ 467,04$ & $\mathrm{R} \$ 467,04$ \\
\hline Hemograma & $100 \%$ & 4 & $\mathrm{R} \$ 13,32$ & $R \$ 53,28$ \\
\hline Glicose & $100 \%$ & 4 & $R \$ 6,00$ & $R \$ 24,00$ \\
\hline Ureia & $100 \%$ & 4 & $R \$ 6,00$ & $R \$ 24,00$ \\
\hline Creatinina & $100 \%$ & 4 & $R \$ 6,00$ & $R \$ 24,00$ \\
\hline Sódio & $100 \%$ & 4 & $R \$ 6,00$ & $R \$ 24,00$ \\
\hline Potássio & $100 \%$ & 4 & $R \$ 6,00$ & $R \$ 24,00$ \\
\hline Transaminase glutâmico-pirúvica (ALT) & $100 \%$ & 4 & $\mathrm{R} \$ 11,04$ & $R \$ 44,16$ \\
\hline Transaminase glutâmico-oxalacética (AST) & $100 \%$ & 4 & $\mathrm{R} \$ 11,04$ & $\mathrm{R} \$ 44,16$ \\
\hline Fosfatase alcalina & $100 \%$ & 4 & $\mathrm{R} \$ 11,04$ & $\mathrm{R} \$ 44,16$ \\
\hline Gama GT & $100 \%$ & 4 & $\mathrm{R} \$ 11,04$ & $\mathrm{R} \$ 44,16$ \\
\hline Bilirrubinas & $100 \%$ & 4 & $R \$ 6,00$ & $R \$ 24,00$ \\
\hline Tempo de protrombina & $50 \%$ & 4 & $R \$ 8,73$ & $\mathrm{R} \$ 17,46$ \\
\hline Radiografia de tórax (posteroanterior e perfil) & $100 \%$ & 4 & $\mathrm{R} \$ 51,74$ & $R \$ 206,96$ \\
\hline Tomografia computadorizada de tórax & $100 \%$ & 4 & $\mathrm{R} \$ 519,72$ & $R \$ 2.078,88$ \\
\hline Tomografia computadorizada de abdome & $100 \%$ & 4 & $R \$ 791,27$ & $R \$ 3.165,08$ \\
\hline Tomografia computadorizada de crânio & $100 \%$ & 1 & $\mathrm{R} \$ 459,18$ & $R \$ 459,18$ \\
\hline RNM de tórax & $30 \%$ & 1 & $\mathrm{R} \$ 1.025,77$ & $\mathrm{R} \$ 307,73$ \\
\hline USG de tórax & $30 \%$ & 1 & $R \$ 92,16$ & $R \$ 27,65$ \\
\hline Cintilografia óssea com Tc99 & $100 \%$ & 2 & $R \$ 312,14$ & $\mathrm{R} \$ 624,27$ \\
\hline PET-TC & $100 \%$ & 1 & $R \$ 969,13$ & $\mathrm{R} \$ 969,13$ \\
\hline Procedimento cirúrgico & $100 \%$ & 1 & $R \$ 8.415,59$ & $\mathrm{R} \$ 8.415,59$ \\
\hline Radioterapia paliativa (por campo) & $70 \%$ & 6 & $R \$ 574,03$ & $R \$ 2.410,91$ \\
\hline Transfusão sanguínea & $100 \%$ & 6 & $R \$ 3.148,80$ & $\mathrm{R} \$ 18.892,80$ \\
\hline Oxigenoterapia mensal & $50 \%$ & 5.475 & $\mathrm{R} \$ 11,00$ & $R \$ 30.112,50$ \\
\hline Infusão (cateter) & $50 \%$ & 12 & $\mathrm{R} \$ 72,84$ & $\mathrm{R} \$ 437,04$ \\
\hline Procedimentos intra-hospitalares & $\%$ uso & Quantidade & Custo unitário & Custo total \\
\hline Hospitalizações imprevistas & $15 \%$ & 12 & $R \$ 447,94$ & $R \$ 806,29$ \\
\hline Visitas à UTI & $8 \%$ & 12 & $\mathrm{R} \$ 1.817,50$ & $\mathrm{R} \$ 1.744,80$ \\
\hline Visitas à emergência & $20 \%$ & 12 & $R \$ 460,06$ & $\mathrm{R} \$ 1.104,14$ \\
\hline TOTAL & & & & $\mathrm{R} \$ 73.682,16$ \\
\hline
\end{tabular}

RNM: ressonância nuclear magnética; USG: ultrassonografia; PET-TC: tomografia computadorizada por emissão de pósitrons; UTI: unidade de terapia intensiva.

\section{Análise de dados}

A quantificação da incerteza envolvida no modelo econômico desenvolvido e a identificação das variáveis que mais afetam essa incerteza são fundamentais para suportar a tomada de decisão. Foi realizada uma análise de sensibilidade do tipo probabilística para validar os resultados dessa avaliação, que considera variações de diversos parâmetros por vez.
Todos os parâmetros da análise foram variados de acordo com a distribuição apropriada (normal, beta ou gama) para cada item. A análise de sensibilidade probabilística foi calculada com 1.000 iterações.

Foi utilizado um limite de disposição a pagar de $\mathrm{R} \$ 86.268$ por PFLY, por QALY e por LY, equivalente a três vezes o Produto Interno Bruto (PIB) per capita nacional, no ano de 2015 [Instituto Brasileiro de Geografia e Estatística (IBGE), 2015]. 
Tabela 5. Custos por eventos adversos

\begin{tabular}{lc}
\hline Evento & Custo por episódio \\
\hline Diarreia & $R \$ 2.113,51$ \\
\hline Rash e acne & $R \$ 286,72$ \\
\hline Fadiga & $R \$ 645,18$ \\
\hline Anemia & $R \$ 15.158,61$ \\
\hline Neutropenia & $R \$ 1.053,63$ \\
\hline Neutropenia febril & $R \$ 17.062,45$ \\
\hline
\end{tabular}

Os resultados foram avaliados e classificados em: quadrante 1 (efetividade incremental $>0$ e custo incremental $>$ 0 ); quadrante 2 (efetividade incremental $<0$ e custo incremental $>0$ ); quadrante 3 (efetividade incremental $<0$ e custo incremental $<0$ ) e quadrante 4 (efetividade incremental $>0$ e custo incremental $<0$ ).

\section{Resultados}

\section{Análise de custo-efetividade e custo-utilidade}

Foram calculados os desfechos e custos totais para um horizonte de tempo de sete anos. As Tabelas 6, 7 e 8 apresentam os resultados da comparação de eficácia entre afatinibe e pemetrexede/cisplatina, erlotinibe e gefitinibe, respectivamente, em termos de PFLY, LY e QALY, além dos custos totais durante todo o horizonte da análise.

O uso de afatinibe, em comparação com pemetrexede/ cisplatina, está associado a aumento da sobrevida livre de progressão (0,41 PFLY), aumento da sobrevida $(0,16$ LY) e aumento dos anos de vida ajustados pela qualidade $(0,21$ QALY), com um custo incremental (R\$ 8.549), resultando em uma razão de custo-efetividade incremental (RCEI) de R\$ 20.639/PFLY, R\$ 53.280/LY e R\$ 39.162/QALY.

Em comparação com erlotinibe, o uso de afatinibe está associado a aumento da sobrevida livre de progressão $(0,46$ PFLY), aumento da sobrevida (0,13 LY) e aumento dos anos de vida ajustados pela qualidade (0,20 QALY), com um custo total menor (- R\$21.327).

Em comparação com gefitinibe, o uso de afatinibe está associado a aumento da sobrevida livre de progressão $(0,53$ PFLY), aumento da sobrevida (0,36 LY) e aumento dos anos de vida ajustados pela qualidade (0,33 QALY), com um custo incremental (R\$ 24.890), resultando em uma RCEl de R\$ 46.709/PFLY, R\$ 67.548/LY e R\$ 73.757/QALY.

Tabela 6. Afatinibe versus pemetrexede/cisplatina

\begin{tabular}{|c|c|c|c|}
\hline Desfecho & Afatinibe & Pemetrexede/Cisplatina & Incremental \\
\hline Custo com medicamento & $R \$ 69.813,15$ & $\mathrm{R} \$ 69.187,28$ & $R \$ 625,87$ \\
\hline Custo com atenção à saúde - LP & $\mathrm{R} \$ 11.126,29$ & $\mathrm{R} \$ 7.464,99$ & $R \$ 3.661,30$ \\
\hline Custo com atenção à saúde - PD & $\mathrm{R} \$ 137.669,53$ & $R \$ 131.332,13$ & $\mathrm{R} \$ 6.337,40$ \\
\hline Custo com eventos adversos & $R \$ 612,15$ & $\mathrm{R} \$ 2.687,33$ & $-R \$ 2.075,18$ \\
\hline Custo total & $\mathrm{R} \$ 219.221,12$ & $\mathrm{R} \$ 210.671,73$ & $\mathrm{R} \$ 8.549,39$ \\
\hline QALY [anos (meses)] & $1,72(20,68)$ & $1,50(18,06)$ & $0,21(2,62)$ \\
\hline LY [anos (meses)] & $2,77(33,27)$ & $2,61(31,34)$ & $0,16(1,93)$ \\
\hline PFLY [anos (meses)] & $1,25(15,11)$ & $0,84(10,13)$ & $0,41(4,97)$ \\
\hline RCEI (QALY) & & & $\mathrm{R} \$ 39.162,79$ \\
\hline RCEI (LY) & & & $\mathrm{R} \$ 53.280,74$ \\
\hline RCEI (PFLY) & & & $\mathrm{R} \$ 20.639,36$ \\
\hline
\end{tabular}

Tabela 7. Afatinibe versus erlotinibe

\begin{tabular}{|c|c|c|c|}
\hline Desfecho & Afatinibe & Erlotinibe & Incremental \\
\hline Custo com medicamento & $R \$ 69.813,15$ & $R \$ 65.371,23$ & $R \$ 4.441,92$ \\
\hline Custo com atenção à saúde - LP & $\mathrm{R} \$ 11.126,29$ & $R \$ 7.078,28$ & $R \$ 4.048,01$ \\
\hline Custo com atenção à saúde - PD & $R \$ 137.669,53$ & $\mathrm{R} \$ 167.693,29$ & $-R \$ 30.023,76$ \\
\hline Custo com eventos adversos & $R \$ 612,15$ & $\mathrm{R} \$ 405,02$ & $R \$ 207,14$ \\
\hline Custo total & $\mathrm{R} \$ 219.221,12$ & $\mathrm{R} \$ 240.547,82$ & $-R \$ 21.326,69$ \\
\hline QALY [anos (meses)] & $1,72(20,68)$ & $1,52(18,31)$ & $0,20(2,37)$ \\
\hline LY [anos (meses)] & $2,77(33,27)$ & $2,64(31,74)$ & $0,13(1,53)$ \\
\hline PFLY [anos (meses)] & $1,25(15,11)$ & $0,80(9,61)$ & $0,46(5,50)$ \\
\hline RCEI (QALY, LY, PFLY) & & & Dominante \\
\hline
\end{tabular}


Tabela 8. Afatinibe versus gefitinibe

\begin{tabular}{|c|c|c|c|}
\hline Desfecho & Afatinibe & Gefitinibe & Incremental \\
\hline Custo com medicamento & $\mathrm{R} \$ 69.813,15$ & $R \$ 34.982,14$ & $\mathrm{R} \$ 34.831,01$ \\
\hline Custo com atenção à saúde - LP & $\mathrm{R} \$ 11.126,29$ & $R \$ 6.416,24$ & $R \$ 4.710,05$ \\
\hline Custo com atenção à saúde - PD & $\mathrm{R} \$ 137.669,53$ & $\mathrm{R} \$ 152.620,97$ & $-R \$ 14.951,44$ \\
\hline Custo com eventos adversos & $\mathrm{R} \$ 612,15$ & $R \$ 311,24$ & $\mathrm{R} \$ 300,91$ \\
\hline Custo Total & $R \$ 219.221,12$ & $R \$ 194.330,59$ & $R \$ 24.890,53$ \\
\hline QALY [anos (meses)] & $1,72(20,68)$ & $1,38(16,63)$ & $0,33(4,05)$ \\
\hline LY [anos (meses)] & $2,77(33,27)$ & $2,40(28,85)$ & $0,36(4,42)$ \\
\hline PFLY [anos (meses)] & $1,25(15,11)$ & $0,72(8,71)$ & $0,53(6,39)$ \\
\hline RCEI (QALY) & & & $\mathrm{R} \$ 73.757,26$ \\
\hline RCEI (LY) & & & $\mathrm{R} \$ 67.548,56$ \\
\hline RCEI (PFLY) & & & $\mathrm{R} \$ 46.709,51$ \\
\hline
\end{tabular}

Em comparação com pemetrexede/cisplatina e com gefitinibe, o afatinibe mostrou-se mais eficaz em todos os desfechos. Esses valores foram considerados custo-efetivos ao assumir-se um limite de disposição a pagar equivalente a três vezes o PIB per capita nacional do ano de 2015 (R\$ 86.628) por PFLY, LY e QALY [Instituto Brasileiro de Geografia e Estatística (IBGE), 2015]. Já em comparação com erlotinibe, o afatinibe demostra-se mais eficaz em todos os desfechos, com menor custo de tratamento, caracterizando-se, assim, como dominante em relação ao erlotinibe em todos os desfechos estudados.

\section{Análise de sensibilidade}

Os resultados da análise de sensibilidade probabilística para a comparação de afatinibe e pemetrexede/cisplatina apresentaram 54,9\% das iterações no quadrante 1, em que há maior custo e maior efetividade. O restante das iterações $(45,1 \%)$ localizou-se no quadrante 4, em que há menor custo e maior efetividade. Das iterações no quadrante 1, 67,6\% localizaram-se abaixo do limiar de custo-efetividade definido (R\$ 86.628 por LY, PFLY e QALY).

Os resultados da análise de sensibilidade probabilística para a comparação de afatinibe e erlotinibe apresentaram $33,8 \%$ das iterações no quadrante 1, no qual há maior custo e maior efetividade. O restante das iterações $(66,2 \%)$ localizou-se no quadrante 4, em que há menor custo e maior efetividade. Das iterações no quadrante 1,71,9\% localizaram-se abaixo do limiar de custo-efetividade definido.

Os resultados da análise de sensibilidade probabilística para a comparação de afatinibe e gefitinibe apresentaram $67,5 \%$ das iterações no quadrante 1, no qual há maior custo e maior efetividade. O restante das iterações $(32,5 \%)$ localizou-se no quadrante 4, em que há menor custo e maior efetividade. Das iterações no quadrante 1, 50,8\% localizaram-se abaixo do limiar de custo-efetividade definido.

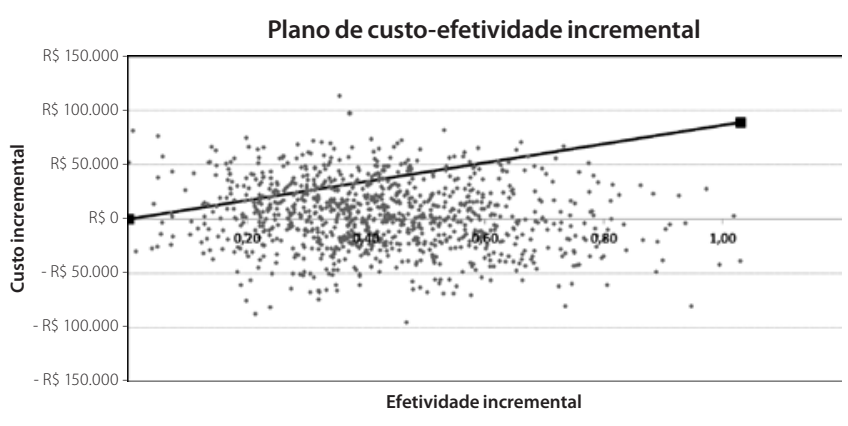

Figura 5. Plano de custo-efetividade incremental (afatinibe versus pemetrexede/cisplatina).

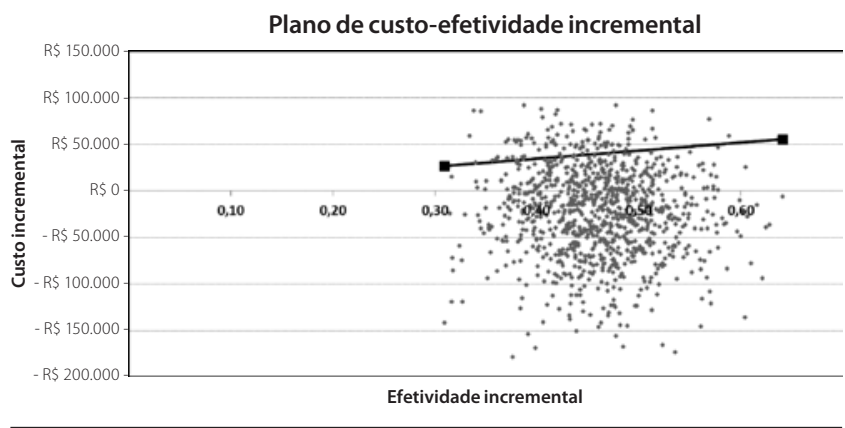

Figura 6. Plano de custo-efetividade incremental (afatinibe versus erlotinibe).

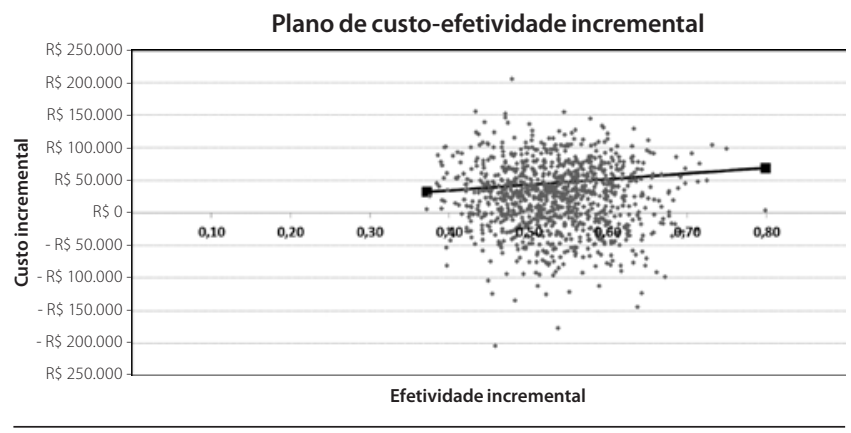

Figura 7. Plano de custo-efetividade incremental (afatinibe versus gefitinibe). 


\section{Discussão}

O objetivo deste estudo foi realizar uma avaliação econômica comparativa entre as estratégias medicamentosas disponíveis para o tratamento de CPNPC, bem como avaliar se o custo adicional proporcionado pelo uso de um medicamento em comparação aos outros é justificado pelo ganho clínico esperado em termos de sobrevida global, sobrevida livre de progressão e qualidade de vida.

Os dados de eficácia foram retirados de ensaios clínicos randomizados e revisões sistemáticas sobre o tratamento medicamentoso de CPNPC com afatinibe, pemetrexede/cisplatina, erlotinibe e gefitinibe, com o objetivo de determinar o impacto dos diferentes tratamentos.

Uma análise de custo-efetividade foi realizada para avaliar o benefício clínico e os custos relacionados à utilização do afatinibe, em comparação ao tratamento com pemetrexede/ cisplatina, erlotinibe e gefitinibe, para pacientes com CPNPC localmente avançado ou metastático com mutação do EGFR, sem tratamento prévio com EGFR-TKI.

O modelo demostrou ganhos em QALY, LY e PFLY para o afatinibe, quando comparado aos demais tratamentos; quando comparado ao erlotinibe, também apresentou economia de recursos.

Para o desfecho de QALY, o afatinibe levou a um aumento de $14,5 \%$, 24,3\% e 12,9\%, em comparação ao pemetrexede/ cisplatina, ao gefitinibe e ao erlotinibe, respectivamente. Já para $L Y$, o aumento foi de 6,1\%, 15,3\% e 4,8\%, em comparação ao pemetrexede/cisplatina, ao gefitinibe e ao erlotinibe, respectivamente. Já o aumento de PFLY foi de 49,0\%, 73,4\% e 57,2\% em comparação ao pemetrexede/cisplatina, ao gefitinibe e ao erlotinibe, respectivamente.

Em relação aos custos, o afatinibe apresentou custos incrementais de $\mathrm{R} \$ \mathbf{8 . 5 4 9 , 3 9}(+4,0 \%), \mathrm{R} \$ 24.890,53(+12,8 \%)$ e - $R \$ 21.326,69$ (-8,9\%), em comparação a pemetrexede/cisplatina, gefitinibe e erlotinibe, respectivamente.

A comparação de afatinibe com pemetrexede/cisplatina demonstrou uma razão de custo-efetividade incremental (RCEI) de R\$ 20.639,36 por PFLY ganho, R\$ 39.162,79 por QALY ganho e R\$ 53.280,74 por LY ganho. Em comparação ao erlotinibe, o afatinibe caracteriza-se como dominante para todos os desfechos, levando a economia de recursos e maior PFLY, LY e QALY. Já na comparação com o gefitinibe, o RCEl foi de R\$ 46.709,51 por PFLY, R\$ 73.757,26 por QALY e R\$ 67.548,56 por LY.

Para avaliar a robustez do modelo, foi realizada uma análise de sensibilidade probabilística, na qual foram simuladas 1.000 iterações. Essa análise apresentou 100\% das iterações, com ganho de PFLY para o afatinibe em comparação aos demais tratamentos, corroborando o ganho de PFLY observado na análise de custo-efetividade. As iterações consideradas custo-efetivas (abaixo do limite de três vezes o PIB per capita de R\$ 86.628, registrado em 2015) foram de $82,2 \%, 90,5 \%$ e $66,8 \%$, respec- tivamente, para a comparação com pemetrexede/cisplatina, erlotinibe e gefitinibe. Foram realizadas análises de sensibilidade para avaliação dos desfechos de QALY e LY, que resultaram em valores compatíveis com os obtidos para o desfecho PFLY, comprovando a robustez dos resultados.

Assim, a análise realizada demonstrou que o uso de afatinibe é custo-efetivo quando comparado aos outros tratamentos medicamentosos disponíveis para pacientes com CPNPC, em termos de LY, QALY e PFLY. Quando comparado ao pemetrexede/cisplatina e gefitinibe, o afatinibe proporciona ganhos em efetividade com maior custo de tratamento. Quando comparado ao erlotinibe, caracterizou-se como dominante, sendo mais eficaz, com menor custo de tratamento.

Cabe ressaltar que essa avaliação econômica tem limitações inerentes à característica do estudo realizado. Por se tratar de um modelo baseado em projeção matemática, temos incertezas associadas. As incertezas e limitações são minimizadas com a aplicação de análises de sensibilidade, que, neste estudo, comprovaram a robustez dos resultados do caso-base. As limitações são inerentes às avaliações econômicas, como a extrapolação dos dados dos estudos clínicos para um horizonte de tempo maior e uso de opinião de especialista para definição e validação de condutas clínicas.

\section{Referências bibliográficas}

Associação Médica Brasileira (AMB). Classificação Brasileira Hierarquizada de Procedimentos Médicos (CBHPM). São Paulo: Associação Médica Brasileira; 2014.

Dietel M, Jöhrens K, Laffert MV, Hummel M, Bläker H, Pfitzner BM, et al. A 2015 update on predictive molecular pathology and its role in targeted cancer therapy: a review focussing on clinical relevance. Cancer Gene Ther. 2015;22(9):417-30.

Fleeman N, Bagust A, McLeod C, Greenhalgh J, Boland A, Dundar Y, et al. Pemetrexed for the first-line treatment of locally advanced or metastatic non-small cell lung cancer. Health Technol Assess. 2010;14 Suppl 1:47-53.

Giotrif [bula]. São Paulo: Boehringer Ingelheim do Brasil Quím. e Farm. Ltda:; 2016. Hirsh V. Next-Generation Covalent Irreversible Kinase Inhibitors in NSCLC: Focus on Afatinib. BioDrugs. 2015;29(3):167-83.

Instituto Brasileiro de Geografia e Estatística (IBGE). PIB per capita. 2015. Disponível em: <http://brasilemsintese.ibge.gov.br/contas-nacionais/ pib-per-capita>

Iressa [bula]. AstraZeneca do Brasil Ltda.; 2011.

Jänne PA, Johnson BE. Effect of epidermal growth factor receptor tyrosine kinase domain mutations on the outcome of patients with non-small cell lung cancer treated with epidermal growth factor receptor tyrosine kinase inhibitors. Clin Cancer Res. 2006;12(14 Pt 2):4416s-20s.

LUX-Lung 7: A Phase Illb Trial of Afatinib(BIBW2992) Versus Gefitinib for the Treatment of 1st Line EGFR Mutation Positive Adenocarcinoma of the Lung. Tabular View. ClinicalTrials.gov. (n.d.). Disponível em: <https://clinicaltrials. gov/ct2/show/record/NCT01466660>. Acessado em: 15 set. 2015.

Miller VA, Hirsh V, Cadranel J, Chen YM, Park K, Kim SW, et al. Afatinib versus placebo for patients with advanced, metastatic non-small-cell lung cancer after failure of erlotinib, gefitinib, or both, and one or two lines of chemotherapy (LUX-Lung 1): a phase 2b/3 randomised trial. Lancet Oncol. 2012;13(5):528-38. 
Ministério da Saúde. Brasil. Câmara de Regulação do Mercado de Medicamentos (CMED). 2016. Disponível em: <http://portal.anvisa.gov. br/cmed>.

Ministério da Saúde (Brasil). Instituto Nacional de Câncer José de Alencar Gomes da Silva (Inca). Estimativa 2014: incidência de câncer no Brasil. Rio de Janeiro: Ministério da Saúde; 2014.

Ministério da Saúde (Brasil). Instituto Nacional de Câncer José Alencar Gomes da Silva (Inca). Estimativa 2016: incidência de câncer no Brasil. Rio de Janeiro: Ministério da Saúde; 2015.

Ministério da Saúde (Brasil). Secretaria de Atenção à Saúde. Carcinoma de pulmão: Portaria SAS/MS n 957, de 26 de setembro de 2014. In: Protocolos Clínicos e Diretrizes Terapêuticas em Oncologia. Brasília: Ministério da Saúde; 2014. p. 171-82.

Park K, Tan E, Zhang L, Hirsh V, O'Byrne K, Boyer M, et al. Afatinib (A) vs gefitinib (G) as first-line treatment for patients (pts) with advanced nonsmall cell lung cancer (NSCLC) harboring activating EGFR mutations: results of the global, randomized, open-label, Phase IIb trial LUX-Lung 7 (LL7). Ann Oncol. 2015;26(Suppl 9):161-2.

Popat S, Mok T, Yang JC, Wu YL, Lungershausen J, Stammberger U, et al. Afatinib in the treatment of EGFR mutation-positive NSCLC--a network meta-analysis. Lung Cancer. 2014;85(2):230-8.

Rosell R, Carcereny E, Gervais R, Vergnenegre A, Massuti B, Felip E, et al.; Spanish Lung Cancer Group in collaboration with Groupe Français de Pneumo-Cancérologie and Associazione Italiana Oncologia Toracica. Erlotinib versus standard chemotherapy as first-line treatment for European patients with advanced EGFR mutation-positive non-smallcell lung cancer (EURTAC): a multicentre, open-label, randomised phase 3 trial. Lancet Oncol. 2012;13(3):239-46.

Sequist LV, Yang JC, Yamamoto N, O'Byrne K, Hirsh V, Mok T, et al. Phase III study of afatinib or cisplatin plus pemetrexed in patients with metastatic lung adenocarcinoma with EGFR mutations. J Clin Oncol. 2013;31(27):3327-34.
Soria JC, Felip E, Cobo M, Lu S, Syrigos K, Lee KH, et al.; LUX-Lung 8 Investigators. Afatinib versus erlotinib as second-line treatment of patients with advanced squamous cell carcinoma of the lung (LUX-Lung 8): an open-label randomised controlled phase 3 trial. Lancet Oncol. 2015;16(8):897-907.

Tarceva [bula]. São Paulo: Produtos Roche Químicos e Farmacêuticos S.A.; 2014.

Wu YL, Zhou C, Hu CP, Feng J, Lu S, Huang Y, et al. Afatinib versus cisplatin plus gemcitabine for first-line treatment of Asian patients with advanced non-small-cell lung cancer harbouring EGFR mutations (LUX-Lung 6): an open-label, randomised phase 3 trial. Lancet Oncol. 2014;15(2):213-22.

Yang JC, Hirsh V, Schuler M, Yamamoto N, O'Byrne KJ, Mok TS, et al. Symptom control and quality of life in LUX-Lung 3: a phase III study of afatinib or cisplatin/pemetrexed in patients with advanced lung adenocarcinoma with EGFR mutations. J Clin Oncol. 2013;31(27):3342-50.

Yang JC, Shih JY, Su WC, Hsia TC, Tsai CM, Ou SH, et al. Afatinib for patients with lung adenocarcinoma and epidermal growth factor receptor mutations (LUX-Lung 2): a phase 2 trial. Lancet Oncol. 2012;13(5):539-48.

Yang JC, Wu YL, Schuler M, Sebastian M, Popat S, Yamamoto N, et al. Afatinib versus cisplatin-based chemotherapy for EGFR mutation-positive lung adenocarcinoma (LUX-Lung 3 and LUX-Lung 6): analysis of overall survival data from two randomised, phase 3 trials. Lancet Oncol. 2015;16(2):141-51.

Zhou C, Wu YL, Chen G, Feng J, Liu XQ, Wang C, et al. Erlotinib versus chemotherapy as first-line treatment for patients with advanced EGFR mutation-positive non-small-cell lung cancer (OPTIMAL, CTONG-0802): a multicentre, open-label, randomised, phase 3 study. Lancet Oncol. 2011;12(8):735-42. 